\title{
Drug-induced Rowell syndrome, a rare and difficult to manage disease: A case report
}

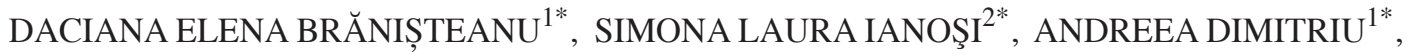 \\ GABRIELA STOLERIU ${ }^{1 *}$, ALEXANDRU OANŢǍ ${ }^{3 *}$ and DANIEL CONSTANTIN BRĂ ${ }^{*}$ ISTTEANU ${ }^{4 *}$ \\ ${ }^{1}$ Department of Dermatology, Grigore T. Popa University of Medicine and Pharmacy, 700715 Iași; \\ ${ }^{2}$ Department of Dermatology, University of Medicine and Pharmacy of Craiova, 200349 Craiova; \\ ${ }^{3}$ Department of Dermatology, Transilvania University of Brașov, 500036 Brasov; ${ }^{4}$ Department of \\ Ophthalmology, Grigore T. Popa University of Medicine and Pharmacy, 700715 Iași, Romania
}

Received November 24, 2016; Accepted May 5, 2017

DOI: 10.3892/etm.2017.5557

\begin{abstract}
Rowell syndrome is defined as the association between lupus erythematosus, erythema multiforme-like lesions and characteristic immunological changes including positive tests for rheumatoid factor, speckled antinuclear antibody, positive anti-Ro or anti-La antibodies. The present report presents the case of a 45-year-old female patient who was previously diagnosed in January 2010 with terbinafine-induced subacute cutaneous lupus erythematosus and was admitted for a skin eruption consisting of erythematous-papular erythema multiforme-like lesions, primarily on the trunk and limbs. The associated symptoms consisted of fatigability, myalgia and gonalgia. In October 2015, the illness reoccurred $\sim 1$ week after the initiation of Helicobacter pylori eradication treatment. Anti-Ro antibodies, rheumatoid factor and antinuclear antibody tests were positive. Given the patient's medical history, clinical manifestations, and laboratory, histopathological and immunofluorescence microscopy findings, a diagnosis of Rowell syndrome was made. Systemic corticosteroids (methylprednisolone; $0.5 \mathrm{mg} / \mathrm{kg} / \mathrm{day}$ ) and immunomodulatory therapy (azathioprine; $50 \mathrm{mg} /$ day) were administered with the associated medication (omeprazole, $20 \mathrm{mg} / \mathrm{day}$; $\mathrm{KCl}, 1 \mathrm{~g} /$ day) and topical dermocorticoids (fluticasone propionate $0.05 \%$ cream; 1 application/day), with a favorable outcome. The major diagnostic criteria for Rowell syndrome are the presence of lupus erythematosus (acute, subacute or systemic), erythema multiforme-like lesions and positive testing for antinuclear antibodies. The minor diagnostic criteria for Rowell syndrome
\end{abstract}

Correspondence to: Professor Simona Laura Ianoşi, Department of Dermatology, University of Medicine and Pharmacy of Craiova, 2 Petru Rareş Street, 200349 Craiova, Romania

E-mail: simonaianosi@hotmail.com

${ }^{*}$ Contributed equally

Key words: lupus erythematosus, erythema multiforme-like, Rowell syndrome are chilblains, the presence of anti-Ro antibodies and positive testing for rheumatoid factor. A diagnosis of Rowell syndrome is made if the patient exhibits all major criteria and at least one minor criterion. The present case met all diagnostic criteria, excluding the presence of chilblains. Notably, in this case there was a co-occurrence of subacute lupus erythematosus and Rowell syndrome lesions, which was drug-induced.

\section{Case report}

The current report presents the case of a 45-year-old female patient who was previously diagnosed in January 2010 at the Dermatological University Clinic (Cluj, Romania) with terbinafine-induced subacute lupus erythematosus (SCLE). The patient received terbinafine $(250 \mathrm{mg} /$ day $)$ for distal subungual onychomycosis of the toenail for 6 months and after 2 months of treatment she developed specific lesions for SCLE. The patient received systemic treatment with antimalarial drugs (hydroxychloroquine; $250 \mathrm{mg} /$ day), corticosteroids (prednisone; $50 \mathrm{mg} /$ day) and immunomodulators (azathioprine; $100 \mathrm{mg} /$ day), and complete remission of lesions was achieved within a year. Although, the course of the disease varied after this. The present study was approved by the Ethics Committee of Grigore T. Popa University of Medicine and Pharmacy (Iași, Romania), and the patient provided written informed consent.

In October 2015, the patient presented, for the first time at our clinic (Clinic of Dermatovenereology, St. Spiridon Emergency Hospital, Iaşi, Romania), with a skin rash consisting of erythematous-papular erythema multiforme-like lesions on the skin, primarily located on the trunk and limbs in (Fig. 1). Onset of illness occurred $\sim 1$ week after the initiation of a Helicobacter pylori (H. pylori) eradication regimen (80 mg/day esomeprasolum and $1 \mathrm{~g} / 12 \mathrm{~h}$ amoxicillin) and subsequently this treatment for $H$. pylori eradication was terminated.

Clinical dermatological examination (Fig. 1) revealed erythematous-edematous macules and plaques, which were well-defined, round and arranged en cocarde, with a tendency to expand and evolve into placards. Certain lesions were solitary, while others evolved into placards. The specific 
lesions were covered by scales that were only adherent to the skin in the center of the lesions and exhibited a pale center. These lesions were accompanied by intense itching and local inflammatory phenomena. In addition, several petechiae were present on the hard palate mucosa. The associated symptoms consisted of fatigability, myalgia and gonalgia. In addition, tests for anti-Ro antibodies and for total antinuclear antibodies using fluorometric enzyme immunoassay (1), and rheumatoid factor using latex-immunoturbidimetry (2) were positive. Serology for herpes infection was performed using the electro-chemiluminescence immunoassay for immunoglobulin G and an IMMULITE 2000 Systems Analyzer (L2KHVG2; Siemens Healthieers, Erlangen Germany.) and ELISA for immunoglobulin $\mathrm{M}$ using a microplate Reader (HSVM.CE, lot 0416; Bio-Rad Laboratories, Inc., Hercules, CA, USA). Both tests were negative.

A skin biopsy was taken for pathological examination and direct immunofluorescence microscopy. The skin biopsy for histopathology was fixed in a buffered $10 \%$ formalin solution for $\sim 12 \mathrm{~h}$, at room temperature. Subsequently, the tissue was embedded in paraffin, cut into $4-\mu \mathrm{m}$ sections, placed on glass microscope slide and stained with hematoxylin and eosin. The slides were analyzed with a light microscope, using a magnification of x40, x100 and x200. Histopathologically, the following features were identified: Areas of atrophied epidermis with exocytosis, vacuolization and foci of necrotic keratinocytes, and a moderate inflammatory perivascular and peridnexal mononuclear infiltrate (Fig. 2A). For the direct immunofluorescence examination, the skin biopsy was immediately transported in cold physiological saline. Section of 4- $\mu \mathrm{m}$ thick obtained at cryotome were fixed with acetone for $15 \mathrm{~min}$ at room temperature. The following antibodies were used: IgA (F031601; dilution 1:20), IgG (F031501; dilution 1:20), IgM (F031701; dilution 1:20), C1q (F025402; dilution 1:4), C3 (F020102; dilution 1:10), Fibrinogen (F011102; dilution 1:60; all from Dako; Agilent Technologies, Inc., Santa Clara, CA, USA). Fluorescence-labeled antibodies were applied to sections and allowed to incubate at room temperature for 30 min. Mounting medium (Dako; Agilent Technologies, Inc.) was applied. Subsequently, using a fluorescence microscope Nikon Eclipse E600, the sites of attachment of the labeled antibodies in the skin were identified at a magnification of $x 40$, x100 and x200. Direct immunofluorescence revealed complement component 3 (C3; Fig. 2B) and immunoglobulin $\mathrm{M}$ in the basement membrane (data not shown).

The patient was monitored periodically (every 6 months) to enable the early detection of potential eye concerns, including orbital and external eye disease, proptosis, enophthalmos, orbital pain, blurred vision, chemosis, restriction of extraocular motility, keratoconjunctivitis sicca, corneal erosion, peripheral corneal infiltration, ulcerative keratitis, interstitial keratitis, endotheliitis, lupus retinopathy, optic neuritis, ischemic optic neuropathy and papilledema, induced by the disease. Furthermore, the potential manifestation of chloroquine maculopathy in response to the administered antimalarial therapy (hydroxychloroquine therapy $200 \mathrm{mg} / \mathrm{day}$; initiated in June 2016 and continued; last follow-up, May 2017) was also monitored.

Based on the patient's history, clinical manifestations (erythema multiforme-like lesions), the antibody detection,

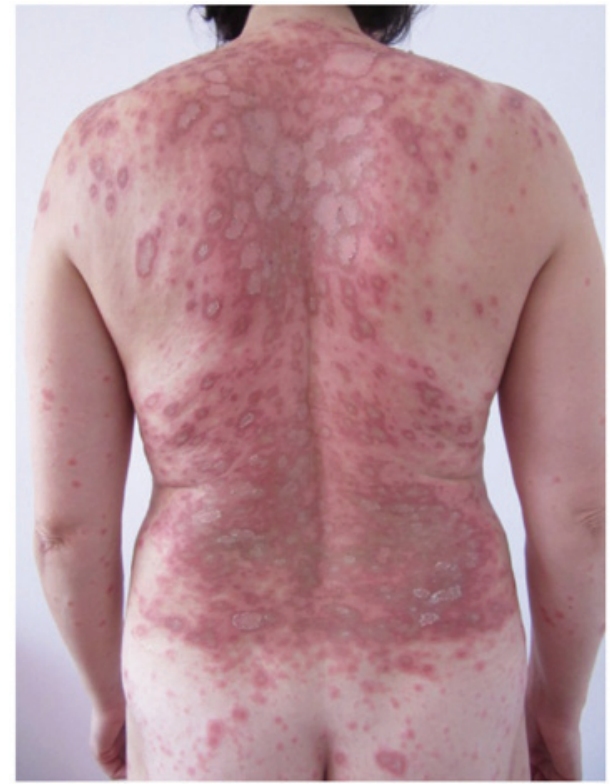

Figure 1. Representative image of the skin lesions observed, en cocarde covered by scales that were only adherent in the center of the lesions and exhibited a pale center on the posterior trunk.
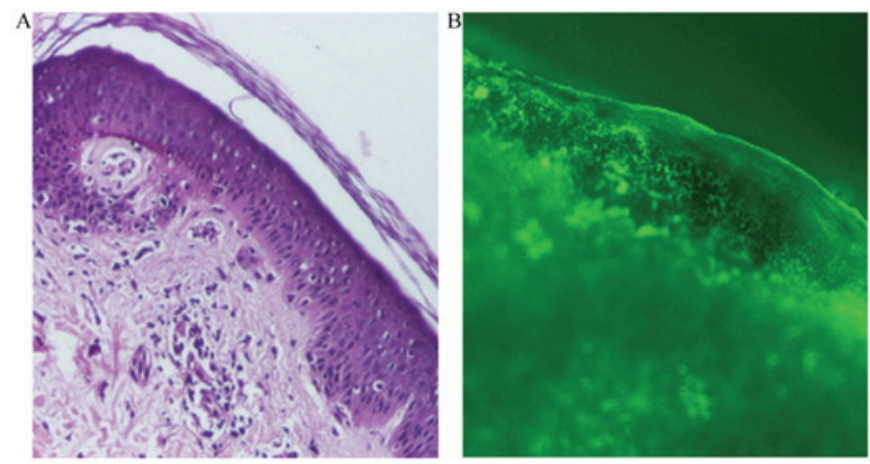

Figure 2. Miscrocopy observation of lesion biopsies. (A) Hematoxylin and eosin staining. Magnification, x100. (B) Positive complement component 3 staining via direct immunofluorescence. Magnification, x100.

histopathological analysis and direct immunofluorescence findings, a diagnosis of Rowell syndrome was made. Therapy with systemic corticosteroids (methylprednisolone; $0.5 \mathrm{mg} / \mathrm{kg}$ ) in combination with immunomodulators (azathioprine; $50 \mathrm{mg} /$ day) was initiated in October 2015, which together with the associated medication (omeprazole, $20 \mathrm{mg} /$ day; $\mathrm{KCl}$, $1 \mathrm{~g} /$ day) and topical dermocorticoids (fluticasone propionate $0.05 \%$ cream; 1 application/day) markedly reduced the patient's symptoms (Fig. 3A). The levels of anti-Ro antibodies, rheumatoid factor and total antinuclear antibodies were monitored periodically, every 3 months.

Given the favorable outcome, the dose of methylprednisolone was gradually reduced with a reduction of $4 \mathrm{mg} / \mathrm{month}$ while maintaining the dose of $100 \mathrm{mg} /$ day of azathioprine. When a dose of $8 \mathrm{mg} /$ day methylprednisolone was reached in May 2016, the patient noticed the reoccurrence of erythema multiforme-like lesions on their trunk, upper limbs and face (Fig. 3B). Therefore, the dose of methylprednisolone was increased to $12 \mathrm{mg} /$ day in June 2016. The course of the 


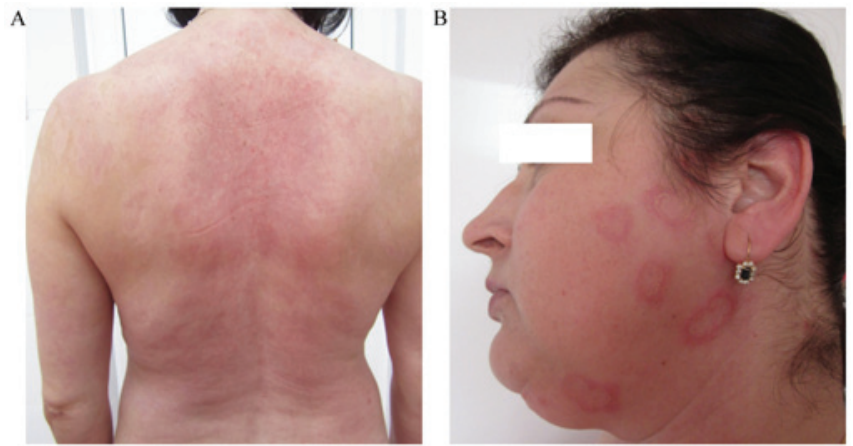

Figure 3. Favorable outcome following treatment and subsequent relapse. (A) Skin lesions on the posterior trunk improved. (B) Relapse of the lesions en cocarde on the face.

disease varied after this, without total control of the skin lesions; however, the patients remained in good general health. Furthermore, hydroxychloroquine (200 mg/day) was added to the treatment regime (June 2016), which reduced the lesions, but did not achieve complete remission. The treatment with dapsone (100 mg/day) was initiated in October 2016 in association with azathioprine (50 mg/day) and hydroxychloroquine $(200 \mathrm{mg} / \mathrm{day})$. The patient decided to terminate all treatments in December 2016 because she developed notable digestive manifestations (nausea and vomiting). At that time (December 2016), all cutaneous lesions were in complete remission until today (last follow-up, May 2017), but arthalgia, myalgia and immunological abnormalities persisted. From the onset of the disease (October 2015) until present (May 2017), the levels of anti-Ro antibodies varied from 44.8 to $46.8 \mathrm{UI} / \mathrm{ml}$, the levels of rheumatoid factor varied from 17 to $16.32 \mathrm{UI} / \mathrm{ml}$ and the total antinuclear antibodies varied from 5.53 to 1.6. In February 2017, the patient persisted with arthalgia, myalgia and asthenia and therefore the hydroxycholoroquine $(200 \mathrm{mg} / \mathrm{day})$ was reinitiated. Furtherore hydroxycholoroquine was also administered for photoprotection reasons and for the prevention of relapse. The patient remains under clinical surveillance, with follow-ups every 6 months.

\section{Discussion}

Rowell syndrome is characterized by the coexistence of lupus erythematosus, erythema multiforme-like lesions and characteristic immunological changes (positive tests for antinuclear antibodies, rheumatoid factor and anti-Ro/anti-La antibodies) (3-11). Until 2014, 37 cases of Rowell syndrome were reported in the literature according to the data published by Bhat et al (4). The first description of this association was made by Scholtz in 1922 (6). In 1963, Rowell et al (7) defined a syndrome consisting of discoid lupus erythematosus, erythema multiforme-like lesions and immunological abnormalities, including the presence of rheumatoid factor and antinuclear antibodies. In 1995, Lee et al (9) suggested the inclusion of chilblains (pernio) to the diagnostic criteria for Rowell syndrome.

In 2000, Zeitouni et al (10) proposed major and minor criteria for the diagnosis of Rowell syndrome. The major criteria were as follows: The simultaneous presence of lupus erythematosus (acute, subacute or systemic), erythema multiforme-like lesions and antinuclear antibodies. The minor criteria were the presence of chilblains, anti-Ro/anti-La antibodies and rheumatoid factor. A positive diagnosis of Rowell syndrome is made in the presence of all major criteria and at least one minor criterion.

An analysis of the 18 case reports of Rowell syndrome diagnosed from 1963-2000 (11) revealed that total antinuclear antibody positivity is the most important feature for the diagnosis of Rowell syndrome, occurring in $88 \%$ of cases, of which $53 \%$ were anti-Ro/anti-La antibodies. Rheumatoid factor was present in $41 \%$ of cases $(4,11)$. The immunologic abnormalities observed in Rowell syndrome are also observed in subacute cutaneous lupus erythematosus (SCLE) (4).

In recent years, the definition of Rowell syndrome has become controversial, with certain researchers suggesting that the association of lupus erythematosus and erythema multiforme may be a coincidence (12), an overlapping syndrome $(4,12)$, a different variant of cutaneous lupus erythematosus, a subtype of chronic lupus erythematosus or an independent subtype of lupus erythematosus $(4,12)$.

Classic erythema multiforme is triggered by various factors, including infectious agents (Mycoplasma pneumonia and herpes simplex virus), medicines (antibiotics, anticonvulsants, nonsteroidal anti-inflammatory drugs and tuberculostatic drugs), malignancies and diseases of the connective tissue $(6,13)$. Erythema multiforme is not associated with specific autoimmune abnormalities $(3,6)$.

Subacute lupus erythematosus (SCLE) is a specific form of lupus erythematosus, in which patients primarily exhibit cutaneous manifestations and typically have a good prognosis $(14,15)$.SCLE is an autoimmune disease (16) characterized by the excessive production of autoantibodies by activated $\mathrm{B}$ cells and autoreactive T cells $(17,18)$. SCLE is characterized by annular or psoriasiform cutaneous lesions located above the waist (neck, trunk and outer aspect of the arms) (14). The following etiologic factors are associated with SCLE: Genetic predisposition (human leukocyte antigen-DR3); immunological factors $(60 \%$ of cases are positive for total antinuclear antibodies, of which $80 \%$ of cases are associated with the presence of anti-Ro antibodies; environmental factors (exposure to ultraviolet radiation, radiotherapy, psoralen combined with ultraviolet A treatment and certain drugs, including thiazide diuretics, calcium blockers, statins, inhibitors of angiotensin converting enzyme, terbinafine and griseofulvin) (15,19-22); and an epigenetics study has reported that autoreactive $\mathrm{T}$ cells and B cells in patients with SCLE have evidence of altered patterns of DNA methylation, modifications of histones and microRNA) (23).

The clinical and histological differentiation of discoid lupus erythematosus from erythema multiforme is challenging. The early skin lesions observed in SCLE are annular and polycyclic, and may resemble erythema multiforme. Also, the necrotic keratinocytes that are frequently present in erythema multiforme can be present in SCLE $(3,6)$. In the early stages of erythema multiforme, there may be a swelling of the endothelial cells and perivascular mononuclear infiltration, followed by hydropic degeneration, keratinocyte necrosis and subepidermal bubbling $(12,13)$. SCLE is characterized by the presence of hyperparakeratosis with follicular plugs, vacuolar changes at the dermoepidermal junction, apoptotic 
keratinocytes, thinning of the basal membrane, and perivascular and periadnexal lymphocytic infiltrate $(15,24)$.

Anatomopathologically, Rowell syndrome and erythema multiforme are similar, with the presence of necrotic keratinocytes (12). In terms of direct immunofluorescence, Rowell syndrome and erythema multiforme exhibit similar finding, including deposits of immunoglobulin $\mathrm{M}$ and $\mathrm{C} 3$ in the dermal capillaries in the early stage, followed by granular $\mathrm{C} 3$ deposits along the dermoepidermal junction (12). Deposits of immunoglobulin $\mathrm{A}, \mathrm{G}$ and $\mathrm{M}$, and $\mathrm{C} 3$, at the dermoepidermal junction have been identified in $60 \%$ of patients with SCLE (12). Lupus erythematosus and Rowell syndrome respond to the same therapeutic regimen, including azathioprine, antimalarial drugs (particularly hydroxychloroquine), prednisone, dapsone and cyclosporine $(3,12)$. Response to treatment is variable and frequent recurrences have been reported (12).

The case discussed in the present reports meets all the major and minor diagnostic criteria for Rowell syndrome, excluding the presence of chilblains. The patient discusse in the present study did not present with Raynaud syndrome, erythema multiforme-like palmar-plantar involvement or bullous lupus erythematosus lesions, as other cases of Rowell syndrome reported in the literature have $(11,24)$. The present case was not preceded by signs of respiratory infection or herpes simplex virus type I or II lesions, and serology for herpes infection was negative. Notably, in the present case a drug was the triggering factor for SCLE and Rowell syndrome lesions. The first SCLE episode was triggered by terbinafine, for which adverse effects have previously been reported in the literature (25). The course of the disease in the present study was favorable under treatment, with complete remission after $\sim 1$ year. However, after a 4-year-long interval, the SCLE reoccurred with the erythema multiforme-like lesions, this time shortly after the introduction of treatment to eradicate H. pylori infection.

The cases of Rowell syndrome reported in the literature had a good prognosis with complete remission of skin lesions within 1 year. In the present study, although the patient entered remission for $\sim 1$ year initially, reoccurrence of the disease occurred after a 4-year-long interval and the interval to complete remission of lesions was double in the present study ( $\sim 2$ years). In addition, in order to avoid the fluctuating course with relapse of the Rowell syndrome, proactive treatment with hydroxychloriquine $200 \mathrm{mg} /$ day was introduced throughout the summer months of the year. Furthermore, in the reported case, the Rowell syndrome was drug-induced. In addition, the typical Rowel syndrome skin lesions, the patient continued to present with high levels of anti-Ro antibodies, rheumatoid factor and total antinuclear antibodies, though at much lower levels compared with at onset. The high levels of these antibodies whilst under therapy highlights the requirement for surveillance in Rowell syndrome, in addition to the long-term and unpredictable course of the disease. Different therapies studied for Rowell syndrome have produced variable results $(4,11,12)$, highlighting the importance of developing novel and more effective treatments. However, a more accurate understanding of the pathophysiology of Rowell syndrome, in addition to a consensus classification of Rowell syndrome as a distinct entity or a particular form of lupus erythematosus/erythema multiforme is required.

\section{References}

1. Kumar Y, Bhatia A and Minz RW: Antinuclear antibodies and their detection methods in diagnosis of connective tissue diseases: A journey revisited. Diagn Pathol 4: 1, 2009.

2. Borque L, Barozzi D and Ferrari L: The determination of rhematoid factors by an immunoturbidimetric assay on boehringer mannheim/hitachi analysis systems. Klin Lab 40: 445-453, 1994.

3. Yachoui R and Cronin P: Systemic lupus erythematosus associated with erythema multiforme-like lesions. Case Rep Rheumatol 2013: 212145, 2013.

4. Bhat RY, Varna C, Bhatt S and Balachandran C: Rowell syndrome. Indian Dermatol Online J 5 (Suppl 1): S33-S35, 2014.

5. Aydin F, Senturk N, Yuksel EP, Yildiz L, Canturk T and Turanli AY: Systemic lupus erythematosus with an erythema multiforme-like lesions. Indian J Dermatol 52: 56-58, 2007.

6. Solanki D, Dalal E and Darji N: Case report of Rowell's Syndrome. Int J Sci Res 3: 7-8, 2014.

7. Rowell NR, Beck JS and Anderson JR: Lupus eythematosus and erythema multiforme-like lesions. A syndrome with characteristic immunological abnormalities. Arch Dermatol 88: 176-180, 1963.

8. Antiga E, Caproni M, Bonciani D, Bonciolini V and Fabbri P: The last word on the so-called 'Rowell's syndrome'? Lupus 21: 577-585, 2012.

9. Lee S, Schloss E and Kowichi J: Rowell's syndrome: A case report with subacute cutaneous lupus erythematosus and erythema multiforme. Can J Dermatol 7: 807-810, 1995.

10. Zeitouni NC, Funaro D, Cloutier RA, Gagné E and Claveau L: Redefining Rowell's syndrome. Brit J Dermatol 142: 343-346, 2000.

11. Lee A, Batra P, Furer V, Cheung W, Wang N and Franks A Jr: Rowell syndrome (systemic lupus erythematosus+erythema multiforme). Dermatol Online J 15: 1, 2009.

12. Andronache IT, Suta C, Ionescu C, Calistrat A and Suta M: Rowell syndrome-a controversial clinical entity. Rom J Rhematol 24: 230-234, 2015.

13. Creţu A, Dimitriu A, Brănişteanu D and Brinişteanu DE: Erythema multiforme-etiopathogenic, clinical and therapeutic aspects. Rev Med Chir Soc Med Nat Iasi 119: 55-61, 2015.

14. Wolff K, Goldsmith LA, Katz S, Gilchrest BA, Paller AS and Leffell D (eds): Fitzpatrik's Dermatology in General Medicine. 7th edition. USA, pp376-384, 2008.

15. Branisteanu DE, Labontu A, Ciobanu D, Stoleriu G, Branisteanu DC and Oanta A: Possible progression of subacute lupus erythematosus-case report. Rev Med Chir Soc Med Nat 118: 381-386, 2014.

16. Gu Y, Zhu T, Wang Y and Xu H: Systemic lupus erythematosus with intestinal perforation: A case report. Exp Ther Med 10: 1234-1238, 2015.

17. Zhang B, Shi Y and Lei TC: Detection of active P-glucoprotein in systemic lupus erythematosus patients with poor disease control. Exp Ther Med 4: 705-710, 2012.

18. Rao L, Liu G, Li C, Li Y, Wang Z, Zhou Z, Tong S and Wu X: Specificity of anti-SSB as a diagnostic marker for the classification of systemic lupus erythematosus. Exp Ther Med 5: 1710-1714, 2013.

19. Saurat JH, Lachapelle JM, Lipsker D and Thomas D: Dermatologie et infections sexuellement transmissibles. 5th edition. Ed Tsunami, France, PP346-353, 2009.

20. Burns T, Breathnach S, Copz N and Griffiths C (eds): Rook's Textbook of Dermatology, eighth edition, Blackwell Publishing, USA, 51: 2, 2010.

21. Zhu X, Li F, Yang B, Liang J, Qin $\mathrm{H}$ and $\mathrm{Xu} \mathrm{J}$ : Effects of ultraviolet $\mathrm{B}$ exposure on DNA methylation in patients with systemic lupus erythematosus. Exp Ther Med 5: 1219-1225, 2013.

22. Brănişteanu DE, Molodoi AD, Stătescu L, Petrescu Z, Vasiluţ D, Anisiei E, Ferariu D and Brănişteanu D: Chilblain lupus in an adolescent. Rev Med Chir Soc Med Nat Iasi 112: 646-651, 2008 (In Romanian).

23. Xiao B and Zuo X: Epigenetics in systemic lupus erythematosus. Biomed Rep 4: 135-139, 2016.

24. Kempf W, Hantschke M, Kutzner $\mathrm{H}$ and Burgdorf WHC: Dermatopathology. Steinkopff Verlag, Germany, page 66, 2008

25. Bonsmann G, Schiller M, Luger TA and Ständer S: Terbinafine-induced subacute cutaneous lupus erythematosus. J Am Acad Dermatol 44: 925-931, 2001.

This work is licensed under a Creative Commons Attribution-NonCommercial-NoDerivatives 4.0 International (CC BY-NC-ND 4.0) License. 